\title{
THE AREA UNDER THE ROC CURVE AS A CRITERION FOR CLUSTERING EVALUATION
}

\author{
Helena Aidos ${ }^{1}$, Robert P.W. Duin ${ }^{2}$ and Ana Fred ${ }^{1}$ \\ ${ }^{1}$ Instituto de Telecomunicações, Instituto Superior Técnico, Lisbon, Portugal \\ ${ }^{2}$ Pattern Recognition Laboratory, Delft University of Technology, The Netherlands \\ haidos@lx.it.pt,r.duin@ieee.org,afred@lx.it.pt
}

\begin{abstract}
Keywords: Clustering Validity, Robustness, ROC curve, Area under curve, Semi-supervised
Abstract: In the literature, there are several criteria for validation of a clustering partition. Those criteria can be external or internal, depending on whether we use prior information about the true class labels or only the data itself. All these criteria assume a fixed number of clusters $k$ and measure the performance of a clustering algorithm for that $k$. Instead, we propose a measure that provides the robustness of an algorithm for several values of $k$, which constructs a ROC curve and measures the area under that curve. We present ROC curves of a few clustering algorithms for several synthetic and real-world datasets and show which clustering algorithms are less sensitive to the choice of the number of clusters, $k$. We also show that this measure can be used as a validation criterion in a semi-supervised context, and empirical evidence shows that we do not need always all the objects labeled to validate the clustering partition.
\end{abstract}

\section{INTRODUCTION}

In unsupervised learning one has no access to prior information about the data labels, and the goal is to extract useful information about the structure in the data. Typically, one can apply clustering algorithms to merge data objects into small groups, unveiling their intrinsic structure. Two approaches can be adopted in clustering: hierarchical or partitional (Jain et al., 1999; Theodoridis and Koutroumbas, 2009).

Usually it is hard to evaluate clustering results without any a priori knowledge of the data. Validation criteria proposed in the literature can be divided in external and internal (Theodoridis and Koutroumbas, 2009). In external criteria, like Rand Statistics, Jaccard Coefficient and Fowlkes and Mallows Index (Halkidi et al., 2001), one has access to the true class labels of the objects. Internal criteria are based on the data only, such as the average intra-cluster distance or the distance between centroids. Silhouette, DaviesBouldin and Dunn indexes (Bolshakova and Azuaje, 2003) are examples of these measures.

There are some drawbacks in using either type of criteria. To use external criteria we need to have the true class label for each object, given by an expert, and this is not always possible or practical. We might have only labels for a small part of the entire dataset. On the other hand, using internal criteria might give a wrong idea of a good clustering. Since internal criteria are based on intra and/or inter cluster similarity, these criteria may be biased towards one clustering algorithm relative to another one. So, when possible, an external criterion is preferable since all the clustering algorithms are equally evaluated.

In the literature, external and internal criteria are designed for the evaluation of clustering algorithms for a fixed number of clusters, $k$. In this paper, we propose to use a ROC curve and the area under that curve (AUC) to study the robustness of clustering algorithms for several values of $k$, instead of a fixed $k$. Also, we study the advantages of using this measure when only a few objects are labeled.

\section{THE PROPOSED CRITERION}

A ROC (Receiver Operating Characteristic) curve is normally used in telecommunications; it is also used in medicine to evaluate diagnosis tests. In machine learning, this curve has been used to evaluate classification methods (Bradley, 1997). Here we will use it to evaluate the robustness of clustering algorithms.

Let $C=\left\{C_{1}, \ldots, C_{k}\right\}$ be a partition of a dataset $X$ obtained by a clustering algorithm and $\mathcal{P}=$ $\left\{P_{1}, \ldots, P_{m}\right\}$ be the true labeling partition of the data. 

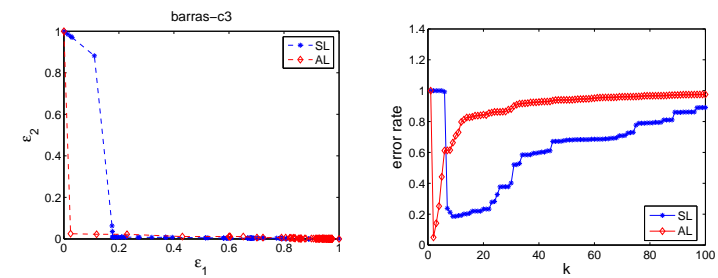

Figure 1: Synthetic dataset with two clusters. Top: ROC curve for single-link (SL) and average-link (AL). Bottom: Error rate for SL and AL, which corresponds to the sum of the two type of errors, $\varepsilon_{1}$ and $\varepsilon_{2}$.

\subsection{ROC Curve}

In this paper, a ROC curve shows the fraction of false positives out of the positives versus the fraction of false negatives out of the negatives. Consider two given points $\mathbf{x}_{a}, \mathbf{x}_{b}$; a type I error occurs if those two points are clustered separated when they should be in the same cluster, i.e., for any pair of objects $\left(\mathbf{x}_{a}, \mathbf{x}_{b}\right)$, type I error is given by $\varepsilon_{1} \equiv P\left(\mathbf{x}_{a} \in C_{i}, \mathbf{x}_{b} \in\right.$ $\left.C_{j} \mid \mathbf{x}_{a}, \mathbf{x}_{b} \in P_{l}\right), i \neq j$ and type II error is given by $\varepsilon_{2} \equiv P\left(\mathbf{x}_{a}, \mathbf{x}_{b} \in C_{i} \mid \mathbf{x}_{a} \in P_{j}, \mathbf{x}_{b} \in P_{l}\right), j \neq l$. In terms of the ROC curve, for a clustering algorithm with varying $k$, for each $k$ we compute the pair $\left(\varepsilon_{1}^{k}, \varepsilon_{2}^{k}\right)$, and we join those pairs to get the curve (see figure 1 top).

We define that a clustering partition $C$ is concordant with the true labeling, $\mathcal{P}$, of the data if

$$
\begin{cases}\varepsilon_{1}=0 & \text { if } k \leq m \\ \varepsilon_{2}=0 & \text { if } k \geq m \\ \varepsilon_{1}=\varepsilon_{2}=0 & \text { if } k=m .\end{cases}
$$

We call a ROC curve proper if, when varying $k$, $\varepsilon_{1}$ increases whenever $\varepsilon_{2}$ decreases and vice-versa. These increases and decreases are not strict. Intuitively, small values of $k$ should yield low values of $\varepsilon_{1}$ (at the cost of higher $\varepsilon_{2}$ ) if the clustering algorithm is working correctly. Similarly, large values of $k$ should lead to low values of $\varepsilon_{2}$ (at the cost of higher $\varepsilon_{1}$ ).

\subsection{Evaluate Robustness}

At some point, a clustering algorithm can make bad choices: e.g., an agglomerative method might merge two clusters that in reality should not be together. Looking at the curve can help in predicting what is the optimal number of clusters for that algorithm, $k^{\prime}$, which minimizes the error rate; it is given by $k^{\prime}=\arg \min \left(\varepsilon_{1}+\varepsilon_{2}\right)$. In figure 1 , right, we plot the sum of the two types of errors as a function of the number of clusters, $k$; this is equivalent to the error rate. In figure 1 left, we see a knee in the curves which corresponds to the lowest error rate found in the bottom plot. We see that average-link (AL) merges clusters correctly to obtain the lowest error rate when the true number of clusters is reached $(k=2)$. On the other hand, for single-link (SL), the minimum error rate is only achieved when $k=9$. Since that number is incorrect, the minimum of the AL curve is lower (better) than the minimum of the SL curve.

In the previous example, visually inspecting the ROC curve shows that AL performs better than SL: the former's curve is closer to the axes than the latter's. However, visual inspection is not possible if we want to compare several clustering algorithms; we need a quantitative criterion. The criterion we choose is the AUC. A lower AUC value corresponds to a better clustering algorithm, which will be close to the true labeling for some $k$. In the example, we have $A U C=0.0247$ for $\mathrm{AL}$ and $A U C=0.1385$ for SL. Also, if $A U C=0$ then the clustering partition $C$ is concordant with the true labeling, $\mathcal{P}$. This definition is consistent with (1).

The ROC curve can also be useful to study the robustness of clustering algorithms to the choice of $k$. We say that a clustering algorithm is more robust to the choice of $k$ than another algorithm if the former's AUC is smaller than the latter's. In the example, AL is more robust to the choice of $k$ than SL.

\subsection{ROC and Parameter Selection}

Some hierarchical clustering algorithms need to set a parameter in order to find a good partition of the data (Fred and Leitão, 2003; Aidos and Fred, 2011). Also, most of the partitional clustering algorithms have parameters which need to be defined, or are dependent of some initialization. For example, $k$-means is a partitional algorithm that needs to be initialized.

Typically, $k$-means is run with several initializations and the mean of some measure (e.g. error rate) is computed, or the intrinsic criterion (sum of the distance of all points to their respective centroid) is used, to choose the best run. We could also consider a fixed initialization for $k$-means like the one proposed by $(\mathrm{Su}$ and Dy, 2007). In this paper we compute the mean (over all runs) of type I error and type II error to plot the ROC curve for this algorithm.

\subsection{Fully Supervised Case}

In the fully supervised case, we assume that we have access to the labels of all samples and we apply clustering algorithms to that data. The main goal is to study the robustness of each clustering algorithm as described in section 2.2. 


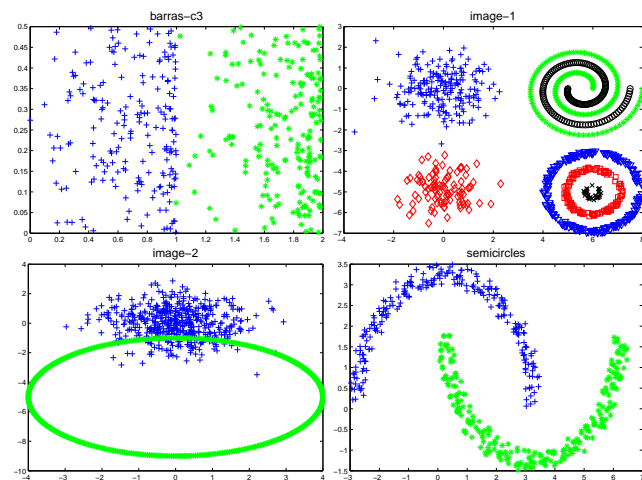

Figure 2: Synthetic datasets.

\subsection{Semi-supervised Context}

We want to study the evolution of the AUC as the fraction of data which has labels becomes smaller. We begin by applying the clustering algorithms to the complete datasets, as in previous section. However, only $10 \%$ of the points are used to compute the ROC curve, and consequently the AUC. The whole dataset is used to perform clustering, whereas the AUC is computed with only a part of the data. This mimics what would happen in a real situation if only part of the data had labels available.

This process is done $M$ times, each time using a different $10 \%$ subset of the data for computing the AUC. This process is run also with $20 \%, 30 \%, \ldots$, $100 \%$ of the points used for the AUC computation.

\section{EXPERIMENTAL RESULTS AND DISCUSSION}

We consider several synthetic (see figure 2) and real datasets, from the UCI machine Learning Repository ${ }^{1}$, to study the robustness of clustering algorithms using the measure described in the previous section. We use 7 traditional clustering algorithms: single-link (SL), average-link (AL), complete-link (CL), Wardlink (WL), centroid-link (CeL), median-link (MeL) and $k$-means (Theodoridis and Koutroumbas, 2009), and two clustering algorithms based on dissimilarity increments: SLAGLO (Fred and Leitão, 2003) and SLDID (Aidos and Fred, 2011).

\subsection{ROC and Parameter Selection}

SLAGLO and SLDID have one parameter that needs to be set (Fred and Leitão, 2003; Aidos and Fred,

\footnotetext{
${ }^{1} \mathrm{http}: / /$ archive.ics.uci.edu/ml
}

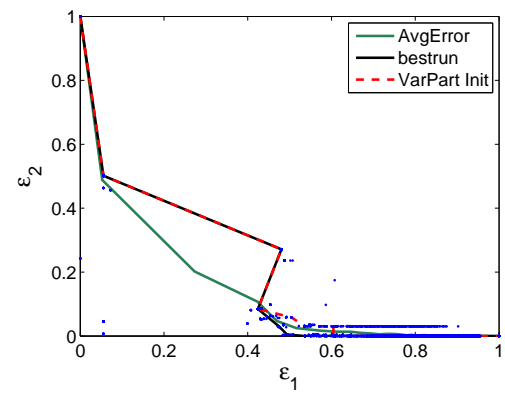

Figure 3: Synthetic dataset with four clusters. Blue dots correspond to the error values for 100 different initializations of $k$-means. AvgError is the ROC curve corresponding to the mean of type I and II errors; bestrun is the ROC curve for the best run according to the intrinsic criterion of $k$-means; VarPart Init is the ROC curve for $k$-means with a fixed initialization based on (Su and Dy, 2007).

2011). As described in section 2.3, we use the AUC to decide the best parameter for each algorithm.

Figure 3 shows the ROC curves for $k$-means, for each strategy described in section 2.3. The figure shows that the curve based on the mean of type I and II errors is proper; the other two are not. This curve also has the lowest AUC. In the following, we plot the ROC curve of $k$-means using several initializations and the mean of the type I and II errors.

\subsection{Fully Supervised Case}

In this section we study the case described in section 2.4. Figure 4 shows the results of applying the clustering algorithm to the datasets described above. For brevity, we present plots only for three of the datasets; we then summarize all the results in table 1.

From table 1 we can see that SLAGLO is more robust in the synthetic data than other clustering algorithms. However, in real datasets, WL and CeL seem to be the best algorithms. In some datasets, we get high values of $\varepsilon_{1}$ and $\varepsilon_{2}$ for some algorithms (such as $\mathrm{CL}$ and $\mathrm{MeL}$ on the cigar dataset) which indicate that these clustering algorithms are not appropriate for that dataset. One of the datasets (crabs) is very hard to tackle for all algorithms.

\subsection{Semi-supervised Case}

As described in section 2.5, we simulate a semisupervised situation to study the advantages of the AUC as a clustering evaluation criterion. In these experiments we use $M=50$ runs.

Figure 5 shows the values of the AUC versus the percentage of points used to compute the AUC. There is considerably different behavior depending on the 

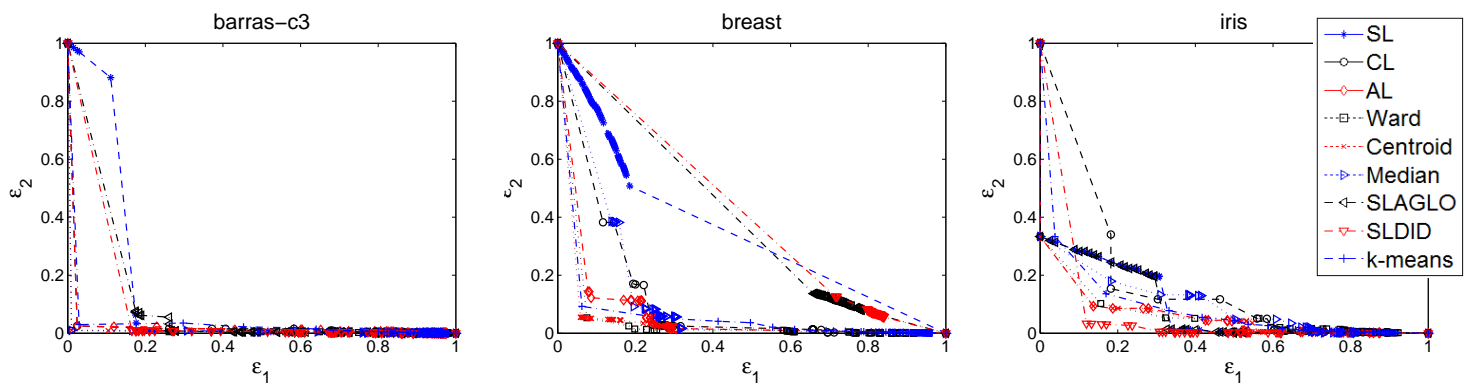

Figure 4: ROC curves for one synthetic datasets and two real datasets.

Table 1: Area under the ROC curve (AUC) when we have access to all labeling of data. Ns is the number of samples, Nf the number of features and $\mathrm{Nc}$ the true number of clusters. The bold numbers are the lowest AUC, which corresponds to the best clustering algorithm.

\begin{tabular}{|l|c|c|c|c|c|c|c|c|c|c|c|c|}
\hline Data & Ns & Nf & Nc & SL & CL & AL & WL & CeL & MeL & SLAGLO & SLDID & $k$-means \\
\hline barras-c3 & 400 & 2 & 2 & 0.139 & 0.025 & 0.025 & $\mathbf{0 . 0 1 0}$ & $\mathbf{0 . 0 1 0}$ & 0.011 & 0.102 & 0.087 & 0.034 \\
image-1 & 1000 & 2 & 7 & 0.007 & 0.082 & 0.068 & 0.063 & 0.067 & 0.087 & $\mathbf{0 . 0 0 2}$ & 0.175 & 0.074 \\
image-2 & 1000 & 2 & 2 & 0.467 & 0.211 & 0.274 & 0.224 & 0.261 & 0.245 & $\mathbf{0 . 0 3 0}$ & 0.343 & 0.266 \\
semicircles & 500 & 2 & 2 & $\mathbf{0}$ & 0.283 & 0.296 & 0.256 & 0.280 & 0.272 & $\mathbf{0}$ & 0.049 & 0.320 \\
austra & 690 & 15 & 2 & 0.489 & 0.428 & 0.472 & 0.470 & 0.483 & 0.486 & 0.489 & 0.496 & $\mathbf{0 . 3 2 0}$ \\
biomed & 194 & 5 & 2 & 0.304 & 0.279 & 0.273 & $\mathbf{0 . 2 5 0}$ & 0.280 & 0.271 & 0.292 & 0.408 & 0.273 \\
breast & 683 & 9 & 2 & 0.351 & 0.121 & 0.070 & $\mathbf{0 . 0 4 9}$ & $\mathbf{0 . 0 4 9}$ & 0.127 & 0.397 & 0.419 & 0.064 \\
chromo & 1143 & 8 & 24 & 0.451 & 0.395 & $\mathbf{0 . 3 9 4}$ & 0.397 & 0.401 & 0.416 & 0.451 & 0.454 & 0.403 \\
crabs & 200 & 5 & 2 & 0.486 & 0.501 & 0.492 & 0.485 & 0.498 & 0.496 & 0.481 & $\mathbf{0 . 4 8 0}$ & 0.499 \\
derm & 366 & 11 & 6 & 0.112 & 0.057 & 0.041 & $\mathbf{0 . 0 3 1}$ & 0.041 & 0.054 & 0.098 & 0.092 & 0.055 \\
ecoli & 272 & 7 & 3 & 0.332 & 0.100 & 0.072 & 0.093 & $\mathbf{0 . 0 7 0}$ & 0.162 & 0.257 & 0.322 & 0.095 \\
german & 1000 & 18 & 2 & 0.488 & 0.492 & 0.485 & 0.484 & 0.489 & $\mathbf{0 . 4 8 1}$ & 0.487 & 0.487 & 0.487 \\
imox & 192 & 8 & 4 & 0.342 & 0.214 & 0.285 & $\mathbf{0 . 0 3 3}$ & 0.325 & 0.140 & 0.148 & 0.195 & 0.134 \\
iris & 150 & 4 & 3 & 0.086 & 0.169 & $\mathbf{0 . 0 5 7}$ & 0.064 & $\mathbf{0 . 0 5 7}$ & 0.097 & 0.083 & 0.067 & 0.089 \\
\hline
\end{tabular}

dataset. For example, in the image-1 dataset the AUC of all algorithms is roughly constant and does not vary much with the percentage of labeled points.

In other datasets we see something very different: in the semicircles and image- 2 datasets some methods have a low AUC for a low percentage of labeled points which then starts to increase with this percentage.

These two different behaviors illustrate an important aspect of the AUC for semi-supervised situations: this measure can become very low for very small percentages of labeled points. In the cases described previously, this is merely a spurious value, since if we had more information (more labeled points) we would find out that the AUC is actually higher.

On the other hand, these plots allow us to decide whether it is worth it to label more data. In general, labeling datasets is expensive; for this reason, it is useful to know if labeling only a subset of data will be enough. One can plot part of the AUC vs. fraction of labeled points curve using the data which is already labeled. If this curve is approximately constant, then it is likely that labeling more data won't bring much benefit. If this curve is rising, then it might be worth considering the extra effort of labeling more data, until one starts seeing convergence in this curve.
There is a further use for these curves. In general, the best way of knowing whether a partition of the data is correct is to know the true partition. In some cases, like in the WL for the imox dataset, the curve is both constant and has a very low value. If one starts investing the time and/or money to label, say, $40 \%$ of the data, one can already be quite sure that the clustering provided by WL is a good one, even without labeling the rest of the data. This is applicable to a few more algorithm-dataset combinations: WL, AL and CeL for derm, or SLDID, AL and WL for iris.

If labeling more data is completely infeasible, the previous reasoning will at least allow researchers to know whether the results obtained on the partiallylabeled data are reliable or not.

\section{CONCLUSIONS}

There are several criteria to evaluate the performance of clustering algorithms. However, those criteria only evaluate clustering algorithms for a fixed number of clusters, $k$. In this paper, we proposed the use of a ROC curve to study the performance of an algorithm for several $k$ simultaneously. This allows measuring 

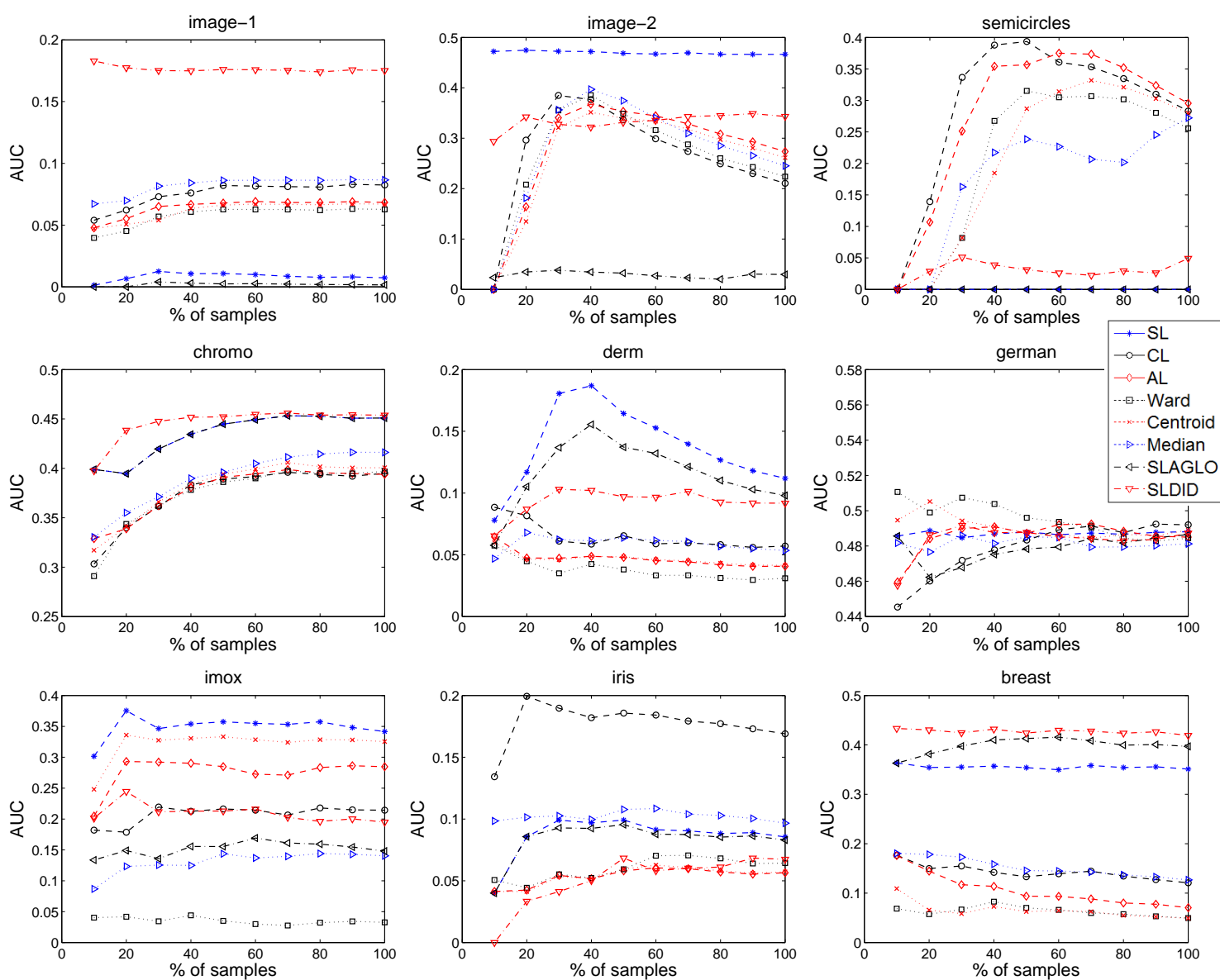

Figure 5: Average of AUC over 50 random subsets of data where we use $\%$ of samples with labels to obtain the ROC curves.

how robust a clustering method is to the choice of $k$.

Moreover, in order to compare the robustness of different clustering algorithms, we proposed to use the area under each ROC curve (AUC).

We showed values of the AUC for fully supervised situations. Perhaps more interestingly, we showed that this measure can be used in semi-supervised cases to automatically detect whether labeling more data would be beneficial, or whether the currently labeled data is already enough. This measure also allows us to extrapolate classes from the labeled data to the unlabeled data, if one can find a clustering algorithm which yields low and consistent AUC value for the labeled portion of the data.

\section{ACKNOWLEDGEMENTS}

This work was supported by the Portuguese Foundation for Science and Technology grant PTDC/EIACCO/103230/2008.

\section{REFERENCES}

Aidos, H. and Fred, A. (2011). Hierarchical clustering with high order dissimilarities. In Proc. of Int. Conf. on Mach. Learning and Data Mining, 280-293.

Bolshakova, N. and Azuaje, F. (2003). Cluster validation techniques for gene expression data. Signal Processing, 83:825-833.

Bradley, A. P. (1997). The use of the area under the roc curve in the evaluation of machine learning algorithms. Patt. Recog., 30(7):1145-1159.

Fred, A. and Leitão, J. (2003). A new cluster isolation criterion based on dissimilarity increments. IEEE Trans. on Patt. Anal. and Mach. Intelligence, 25(8):944-958.

Halkidi, M., Batistakis, Y., and Vazirgiannis, M. (2001). On clustering validation techniques. Journal of Intelligent Information Systems, 17(2-3):107-145.

Jain, A., Murty, M., and Flynn, P. (1999). Data clustering: a review. ACM Comp. Surveys, 31(3):264-323.

Su, T. and Dy, J. G. (2007). In search of deterministic methods for initializing k-means and gaussian mixture clustering. Intelligent Data Analysis, 11(4):319-338.

Theodoridis, S. and Koutroumbas, K. (2009). Pattern Recognition. Elsevier Academic Press, 4th edition. 